\section{Vulnerabilidade programática na prevenção da transmissão materno-fetal da AIDS}

\section{Program deficiencies in the prevention of maternal-fetal transmission of AIDS}

Katia V. de O. Feliciano 1

Maria Helena Kovacs 2

1,2 Coordenação de Pós-graduação. Faculdade de Ciências Médicas da Universidade de Pernambuco.

Rua Arnóbio Marques, 310. Recife, Pernambuco, Brasil.

CEP: 50.100-130. E mail: cpg@ fcm.upe.br

\section{Abstract}

Objectives: to assess the effectiveness of maternalfetal HIV transmission prevention programs at the prenatal care stage.

Methods: a descriptive study was held in the city of Recife, Pernambuco, from May to July 1998, in 27 locations of six Sanitary Districts: 19 Health Centers, two Maternity Clinics and six Family Health Programs (PSF). The study focused on observation and interviews of managers and technical teams. Their work was followed for at least two shifts in randomly selected weekdays, totaling 214 hours. The 355 pregnant women present filled a questionnaire requiring the following information: age, obstetrics history, number of medical visits, accessibility and orientation received.

Results: unmet demand was found in $63,0 \%$ of the institutions. Only $41,6 \%$ of pregnant women being followed had their present medical visit scheduled by the service following the previous one (PSF). In relation to sexually transmitted diseases: all prescribed the Lues Test and 67,0\% summoned sexual partners in case of positive testing. The anti-HIV test was not universally offered and counseling was not provided. Only 26,7\% of the doctors having identified pregnant women at risk of AIDS prescribed the test and 19,8\% of the pregnant women received guidance for sexually transmissible diseases.

Conclusions: institutional vulnerability in the execution of program measures and the broad range of health requirements for women in the reproductive age group emphasizes the need of organizing pre-natal care. Key words Acquired Immunodeficiency Syndrome, Prenatal care, Outcome and process assessment (health care), Program evaluation

\section{Resumo}

Objetivos: avaliar a operacionalidade das ações de prevenção da transmissão materno-fetal do HIV na assistência pré-natal.

Métodos: estudo descritivo realizado na cidade do Recife, Pernambuco, de maio a julho de 1998, em 27 unidades dos seis Distritos Sanitários: 19 Centros de Saúde, duas Maternidades e seis do Programas de Saúde da Família (PSF). A organização foi caracterizada por meio de observações e entrevistas com gerentes e equipes técnicas. Acompanhou-se o processo de trabalho durante, no mínimo, dois turnos, em dias da semana aleatoriamente selecionados, totalizando 214 horas. As 355 gestantes presentes responderam um questionário: idade antecedentes obstétricos, número de consultas, acessibilidade e orientações recebidas.

Resultados: em 63,0\% das unidades houve demanda reprimida. Somente $41,6 \%$ das gestantes em seguimento tiveram a consulta atual agendada pelo serviço após a anterior. Em relação às doenças sexualmente transmissíveis: todos solicitaram o teste luético e 67,0\% convocaram os parceiros quando o exame é positivo; não houve oferta universal do teste anti-HIV, nenhum realizou aconselhamento, apenas $26,7 \%$ dos médicos que identificaram gestantes de risco para AIDS solicitaram o teste e 19,8\% das gestantes receberam orientações sobre essas doenças.

Conclusões: a vulnerabilidade institucional na execução das ações programáticas e o amplo conjunto de necessidades de saúde da mulher em idade reprodutiva reiteram a importância de organizar o pré-natal.

Palavras-chave Síndrome de Imunodeficiência Adquirida, Cuidado pré-natal; Avaliação de processos e resultados (cuidados de saúde), Avaliação de programas 


\section{Introdução}

No ano de 1994, ao mesmo tempo em que o Ministério da Saúde começava a dar prioridade à dimensão organizacional do processo de trabalho voltado para a prevenção e o controle da Síndrome de Imunodeficiência Adquirida (AIDS), fortalecendo o desenvolvimento institucional das Secretarias Estaduais e Municipais com o intuito de melhorar as condições locais de execução do programa, iniciavase o reconhecimento da "feminização" da epidemia que, envolta em um grande silêncio, vinha sendo configurada desde o fim da década de $80.1^{1}$ No Recife, Pernambuco, semelhante à tendência nacional, a razão de sexo, que em 1987 era de 14 homens para uma mulher, chegou a 6:1 em 1991 e passou a ser de 2:1 a partir de 2000. De 1987 a agosto de 2001 foram notificados 54 casos de AIDS em menores de 13 anos de idade, $88,7 \%$ infectados pela via perinatal. A transmissão vertical cresceu ao longo do tempo: inexistente até 1986, era 1,5\% de 1991-1994 e alcançou 2,1\% no período de 1998-2001.2

A tardia resposta institucional, desencadeada em 1995, elegeu a gestação como o momento para diagnosticar a infecção pelo Human Immunodeficiency Virus (HIV) em mulheres, evitando a transmissão do vírus para os seus bebês. Nesse mesmo ano, o Ministério da Saúde divulgou a primeira publicação específica sobre a prevenção da transmissão maternofetal do vírus. Com o avanço da AIDS na população feminina dando origem a novas carências, a partir de 1997, este órgão substituiu a oferta da testagem apenas para mulheres com risco identificado para infecção pelo HIV, pela oferta universal do teste no pré-natal. ${ }^{3}$ Desde então, aos serviços compete solicitar o consentimento verbal da mulher, propiciar o uso da zidovudina para os casos positivos durante a gestação e o parto, fornecer informações para melhorar a qualidade de vida, estimular a prática de sexo seguro, sobretudo, durante a gravidez e o aleitamento materno, orientar sobre o método preferencial de alimentação para os bebês e garantir a assistência ao parto. 4

A análise da assistência pré-natal desenvolvida em Pernambuco, até maio de 1997, utilizando o banco populacional de dados da II Pesquisa Estadual de Saúde e Nutrição, mostra que 82,9\% das mães estudadas tinham feito consulta pré-natal durante a última gravidez - 73,8\% nas famílias mais pobres e $97,9 \%$ nas famílias com maior renda. A cobertura cresce significativamente à medida que aumenta a escolaridade, variando de $63,8 \%$ nas mães analfabetas até $98,2 \%$ naquelas de nível superior. Na Região Metropolitana do Recife, com cobertura pré-natal de 90,3\%, em
$73,5 \%$ dos casos a primeira consulta foi realizada no primeiro trimestre, tendo $1,2 \%$ das gestantes efetuado-a no sétimo mês de gestação, ou mais, e 75,5\% comparecido a cinco ou mais consultas. 5

O aumento da cobertura da atenção pré-natal pode não ter o impacto esperado sobre a transmissão materno-fetal da AIDS, a morbidade e a mortalidade materna e perinatal se persistirem as deficiências comumente encontradas: o início tardio, a falta de qualidade da consulta, a escassez de práticas educativas, o difícil acesso aos recursos de laboratório e os problemas na organização de um sistema articulado de cuidados em níveis hierarquizados de atenção — pré-natal de risco e parto. É preciso considerar que as limitações éticas, humanas e técnicas, que permeiam o atendimento ao parto em geral se acentuam quando da assistência ao parto das gestantes soropositivas. 6

De acordo com a estimativa feita a partir de dados do projeto "Vigilância do HIV por Rede-Sentinela Nacional",3 em 1998 ocorreram no Brasil cerca de 12.898 partos de gestantes infectadas pelo HIV. Dessas, apenas $19,5 \%$ receberam zidovudina injetável no momento do parto, sendo encontrada na região Nordeste a menor proporção de gestantes soropositivas que realizou o tratamento preconizado $(2,6 \%)$. Essas constatações põem em relevo a preocupação com os obstáculos para a organização de uma atenção capaz de responder às necessidades globais da saúde da mulher - sobretudo, quando se tem conhecimento de que as atividades de controle das doenças sexualmente transmissíveis são as menos desenvolvidas no conjunto das práticas direcionadas à população feminina 6 — assim como, com a efetividade das ações programáticas para evitar que as mães transmitam o vírus da AIDS para os seus filhos durante a gravidez e o parto.

Nesse contexto, é a noção de vulnerabilidade que norteia esta avaliação da prevenção da transmissão vertical da AIDS na atenção pré-natal. Proposta por Mann et al.7 para avaliar "suscetibilidade e condições de resposta" em relação ao HIV e à AIDS, 7-,9 esta abordagem utiliza três planos analíticos interdependentes: a) individual — aspectos cognitivos, comportamentais e disponibilidade de ações institucionais para controlar a doença; b) programático ou institucional - os caminhos concretos para satisfazer às necessidades de saúde, promovendo a ligação dos indivíduos com os contextos em que estão inseridos; e c) social — condições de vida, especificidade simbólica do cotidiano, políticas sociais para enfrentar a epidemia e respeito à dignidade $\mathrm{e}$ aos direitos humanos.

Essa maneira de focalizar o problema sugere 
que, do ponto de vista programático, as análises das políticas e das atividades relacionadas com a AIDS contemplem aspectos tais como: a expressão de compromisso por parte dos níveis investidos de poder decisório, a existência de projetos que apóiem a transformação do compromisso em ação, o desenvolvimento de parcerias que viabilizem a operação do modelo de organização do trabalho, o planejamento, a coordenação e o gerenciamento das ações, as respostas às necessidades de prevenção e tratamento, a obtenção de recursos financeiros, as condições para as ações manterem o fluxo de benefícios ao longo do tempo, a conformidade entre o projeto de intervenção e o que está sendo executado e a avaliação do impacto.

Assim, tendo a vulnerabilidade como referencial que fornece sentido prático e objetivo para aferir os sucessos e fracassos das ações, 10 este artigo examina a "operacionalidade" da detecção precoce da infecção pelo HIV em gestantes e da educação preventiva na assistência pré-natal, com o propósito de contribuir para que as mulheres e seus bebês tirem maior proveito da estratégia estabelecida. Levou-se em conta a definição das necessidades sobre as quais intervir, os meios de trabalho, a atuação da equipe em relação aos conteúdos previstos, além da utilização e adequação das distintas alternativas de intervenção em relação às necessidades identificadas.

\section{Métodos}

Estudo descritivo, de corte transversal, realizado para analisar a implementação11,12 da assistência pré-natal nos serviços de atenção primária da Secretaria Municipal de Saúde da Cidade do Recife, Pernambuco, Brasil, destacando as ações voltadas para a transmissão vertical do HIV. As unidades de saúde desta rede operam dois modelos assistenciais: a) tradicional, centrado no atendimento da demanda espontânea da qual capta usuários que recebem algumas ações programáticas; e b) Programa de Saúde da Família (PSF), com atuação em área delimitada onde proporciona atenção integral à saúde de todos os membros de um determinado número de famílias.

A partir das informações sobre ações disponíveis na rede básica de saúde foram identificados: 58 Centros de Saúde (distribuídos nos seis Distritos Sanitários), duas Maternidades (Distritos III e V) e 19 equipes do PSF (exceto no Distrito I), os quais prestam assistência pré-natal. ${ }^{13} \mathrm{Em}$ cada Distrito, o número de Centros de Saúde selecionados é proporcional ao número de serviços desta categoria que desenvolvem o pré-natal, sendo alocados aqueles que, em 1997, apresentaram maior número de consultas na atenção à gestante. Para cada Distrito que conta com PSF, escolheu-se a unidade de maior cobertura. Incluíram-se as Maternidades com atenção pré-natal. A amostra do presente trabalho consta de 27 unidades - 19 Centros de Saúde, duas Maternidades e seis equipes do PSF.

O trabalho de campo foi feito, de maio a julho de 1998, por uma equipe (médica sanitarista, enfermeira e assistente social) com experiência em pesquisa, devidamente treinada. A organização das práticas nesses serviços foi examinada por meio de observação e entrevistas com os gerentes e as equipes técnicas, usando formulário padronizado captação da clientela, modalidades de atendimento, agentes do trabalho, material educativo, convocação de parceiros e apoio diagnóstico. O fluxograma do sistema de acesso à sorologia anti-HIV foi investigado, também, da perspectiva dos profissionais do Laboratório Municipal de Saúde Pública e do Centro de Orientação e Aconselhamento (COA).

Em cada unidade, o trabalho desenvolvido na assistência pré-natal foi observado, no mínimo, durante dois turnos, em dias da semana aleatoriamente selecionados, perfazendo um total de 214 horas, e utilizando formulário próprio - acessibilidade, oferta e fluxograma de atividades, adesão da equipe às padronizações estabelecidas e coleta de material para exame de laboratório. Um questionário foi aplicado a todas as gestantes que aguardavam atendimento - idade, residência, inserção no mercado de trabalho, número de gestações, antecedentes de abortamento, aprazamento da consulta, número de consultas pré-natal e orientações recebidas. Obteve-se uma amostra de 355 gestantes.

A partir da listagem obtida quando da caracterização dos agentes do trabalho, foram identificados 57 médicos, 31 enfermeiras, 12 psicólogas e seis assistentes sociais que realizavam atividades de pré-natal. Para conhecer as percepções relacionadas à execução destas e o acesso a treinamento em doenças sexualmente transmissíveis/AIDS nos últimos dois anos, foram entrevistados 23 médicos, 13 enfermeiras, quatro psicólogas e duas assistentes sociais que estavam prestando atendimento durante as observações.

Esta avaliação foi requerida pelo Programa de Doenças Sexualmente Transmissíveis/AIDS da Secretaria Municipal de Saúde da Cidade do Recife. Os gerentes dos Distritos Sanitários e a direção de cada unidade selecionada autorizaram a sua realização. Para a aplicação dos questionários individuais foi solicitado o consentimento verbal informado e assegurado o caráter espontâneo e confidencial da participação das gestantes e dos profissionais — dois médi- 
cos se recusaram, alegando falta de tempo.

O material de campo foi cuidadosamente revisado e organizado. Para a análise dos resultados, os serviços foram distribuídos de acordo com as características organizacionais - Centros de Saúde, Maternidades e PSF. Na comparação das gestantes atendidas nos três tipos de unidades, estabeleceu-se a existência de diferenças significativas entre a idade das mulheres por meio da análise de variância de Kruskal-Wallis; e aplicou-se o teste do qui-quadrado de tendência para identificar a influência do número de consultas pré-natal no recebimento de orientação. A participação em treinamentos foi avaliada, também, em função da categoria profissional.

\section{Resultados}

Nestas unidades, $48,1 \%$ das mulheres que estão realizando a primeira consulta pré-natal conseguiram a ficha no dia do atendimento $(96,1 \%$ na atenção tradicional). Apenas 41,6\% daquelas que estão em acom- panhamento tiveram a consulta atual agendada pelo serviço após a anterior $(87,0 \%$ das cadastradas no PSF). Em 63,0\% ocorreu rechaço por excesso de demanda, sendo que em $58,8 \%$ destas (todas da atenção tradicional) as mulheres foram orientadas para fazer nova tentativa. A clientela, em geral, necessita chegar muito cedo para conseguir marcar a consulta e espera muito para efetuá-la.

A distribuição etária dessas mulheres mostra uma forte participação das menores de 20 anos (33,0\%), além da elevada concentração no grupo com idade entre 20-34 anos (64,0\%). A amplitude da distribuição inclui gestantes de 13 a 41 anos, não existindo diferença significativa entre as medianas de idade das usuárias dos três tipos de unidade. Somente, $57,5 \%$ residem no mesmo bairro do serviço utilizado (97,1\% no PSF). Cerca de $18,0 \%$ trabalham (62,5\% faltaram ao trabalho para se consultar). Para $45,1 \%$ essa é a primeira gestação e para $14,4 \%$, a quarta ou mais. Dentre as mulheres com duas ou mais gestações, $35,9 \%$ apresentam antecedentes de abortamento (Tabela 1).

Tabela 1

Características sociodemográficas das gestantes, segundo o tipo de unidade. Recife, Pernambuco, Brasil, 1998.

\begin{tabular}{|c|c|c|c|c|c|c|c|c|}
\hline \multirow{3}{*}{ Características } & \multicolumn{8}{|c|}{ Tipo de unidade } \\
\hline & \multicolumn{2}{|c|}{$\begin{array}{c}C S \\
(n=239)\end{array}$} & \multicolumn{2}{|c|}{$\begin{array}{c}\text { MAT } \\
(n=48)\end{array}$} & \multicolumn{2}{|c|}{$\begin{array}{c}\text { PSF } \\
(n=68)\end{array}$} & \multicolumn{2}{|c|}{$\begin{array}{c}\text { Total } \\
(n=355)\end{array}$} \\
\hline & $n$ & $\%$ & $\mathrm{n}$ & $\%$ & $\mathrm{n}$ & $\%$ & $\mathrm{n}$ & $\%$ \\
\hline \multicolumn{9}{|l|}{ Idade (anos)* } \\
\hline$<20$ & 81 & 33,9 & 14 & 29,2 & 24 & 35,3 & 119 & 33,5 \\
\hline $20-34$ & 149 & 62,3 & 34 & 70,8 & 43 & 63,2 & 226 & 63,7 \\
\hline $35 e+$ & 9 & 3,8 & - & - & 1 & 1,5 & 10 & 2,8 \\
\hline \multicolumn{9}{|l|}{ Local de residência } \\
\hline Mesmo bairro da Unidade & 112 & 46,9 & 26 & 54,2 & 66 & 97,1 & 204 & 57,5 \\
\hline Mesmo DS da Unidade & 63 & 26,3 & 10 & 20,8 & 2 & 2,9 & 75 & 21,1 \\
\hline Outro DS & 46 & 19,2 & 8 & 16,7 & - & - & 54 & 15,2 \\
\hline Outro município da RMR & 18 & 7,6 & 4 & 8,3 & - & - & 22 & 6,2 \\
\hline \multicolumn{9}{|l|}{ Inserção no mercado de trabalho } \\
\hline Sim & 50 & 20,9 & 5 & 10,4 & 9 & 13,2 & 64 & 18,0 \\
\hline Não & 189 & 79,1 & 43 & 89,6 & 59 & 86,8 & 291 & 82,0 \\
\hline \multicolumn{9}{|l|}{ Número de gestações } \\
\hline Uma & 108 & 45,2 & 22 & 45,9 & 30 & 44,1 & 160 & 45,1 \\
\hline Duas & 63 & 26,3 & 16 & 33,3 & 14 & 20,6 & 93 & 26,1 \\
\hline Três & 32 & 13,4 & 4 & 8,3 & 15 & 22,1 & 51 & 14,4 \\
\hline Quatro e + & 36 & 15,1 & 6 & 12,5 & 9 & 13,2 & 51 & 14,4 \\
\hline Antecedentes de abortamento** & \multicolumn{2}{|c|}{$(n=131)$} & \multicolumn{2}{|c|}{$(n=26)$} & \multicolumn{2}{|c|}{$(n=38)$} & \multicolumn{2}{|c|}{$(n=195)$} \\
\hline Sim & 49 & 37,4 & 11 & 42,3 & 10 & 26,3 & 70 & 35,9 \\
\hline Não & 82 & 62,6 & 15 & 57,7 & 28 & 73,7 & 125 & 64,1 \\
\hline
\end{tabular}

CS = Centro de Saúde, MAT = Maternidade, PSF = Programa de Saúde da Família, DS = Distrito Sanitário, RMR = Região Metropolina do Recife, * Análise de variância de Kruskal-Wallis $(H=1,172 ; p=0,557)$, ** Excluídas as mulheres de 1 a gestação. 
A pré-consulta, afora cinco Centros de Saúde, é realizada nas outras unidades - em duas pela enfermeira e, em vinte, pela auxiliar de enfermagem constando de tomada de peso e aferição da pressão. Em 59,2\% há delegação de consulta à enfermagem - em três Centros de Saúde, exclusivamente, para quem recebe suplementação alimentar. Nenhum serviço faz pós-consulta. As enfermeiras, no PSF, monitoram a gestação de baixo risco, enquanto na atenção tradicional se restringem à primeira consulta, dando primazia à solicitação dos exames de rotina. A consulta médica está voltada para detecção e controle de fatores de risco (Tabela 2).

Processo de trabalho na atenção pré-natal, segundo o tipo de unidade. Recife, Pernambuco, Brasil, 1998.

\begin{tabular}{|c|c|c|c|c|c|c|c|c|}
\hline \multirow{3}{*}{ Processo de trabalho } & \multicolumn{8}{|c|}{ Tipo de unidade } \\
\hline & \multicolumn{2}{|c|}{$\begin{array}{c}\text { CS } \\
(n=19)\end{array}$} & \multicolumn{2}{|c|}{$\begin{array}{c}\text { MAT } \\
(n=2)\end{array}$} & \multicolumn{2}{|c|}{$\begin{array}{c}\text { PSF } \\
(n=6)\end{array}$} & \multicolumn{2}{|c|}{$\begin{array}{c}\text { Total } \\
(n=27)\end{array}$} \\
\hline & $\mathrm{n}$ & $\%$ & $\mathrm{n}$ & $\%$ & $\mathrm{n}$ & $\%$ & $\mathrm{n}$ & $\%$ \\
\hline Realiza pré-consulta & 14 & 73,7 & 2 & 100,0 & 6 & 100,0 & 22 & 81,5 \\
\hline Delega consulta à enfermagem & 9 & 47,4 & 1 & 50,0 & 6 & 100,0 & 16 & 59,2 \\
\hline Realiza consulta médica & 19 & 100,0 & 2 & 100,0 & 6 & 100,0 & 27 & 100,0 \\
\hline Faz pós-consulta & - & - & - & - & - & - & - & - \\
\hline Executa exame de prevenção de câncer & 19 & 100,0 & 2 & 100,0 & 6 & 100,0 & 27 & 100,0 \\
\hline Solicita sorologia para sífilis & 19 & 100,0 & 2 & 100,0 & 6 & 100,0 & 27 & 100,0 \\
\hline Convoca o parceiro quando o teste luético é positivo & 10 & 52,6 & 2 & 100,0 & 6 & 100,0 & 18 & 66,7 \\
\hline Informa sobre o teste anti-HIV & 9 & 47,4 & 1 & 50,0 & 3 & 50,0 & 13 & 48,1 \\
\hline Realiza aconselhamento pré e pós-teste & - & - & - & & - & & - & - \\
\hline
\end{tabular}

CS = Centro de Saúde, MAT = Maternidade, PSF = Programa de Saúde da Família, HIV = Human Immunodeficiency virus

A Tabela 2 informa, também, que todas as unidades efetuam a prevenção de câncer cérvicouterino - porém, não existe fluxograma para os encaminhamentos internos, tampouco, mecanismos estabelecidos para priorizá-los. Embora o teste luético seja feito como rotina no pré-natal destes serviços, em apenas $66,7 \%$, excetuados nove Centros de Saúde, convida-se o companheiro da gestante com sorologia positiva para comparecer à unidade, para ser avaliado. É habitual, na atenção tradicional, a própria mulher ser responsabilizada pela convocação do parceiro. Nenhum serviço tem aconselhamento pré e pós-teste anti-HIV, mesmo aqueles que dizem informar sobre a testagem $(48,1 \%)$.

O trabalho educativo, quase sempre, é parte integrante da assistência individual. Nas unidades que dizem efetuá-la — todas do PSF, Maternidades e 26,3\% dos Centros de Saúde — as atividades grupais não ocorrem em todos os horários do atendimento pré-natal. No PSF, afora a visita domiciliar do agente comunitário, as mulheres são orientadas para voltar à unidade nos dias previstos para o trabalho coletivo e, na atenção tradicional, os grupos são formados com aquelas presentes na sala de espera. Quando das observações, houve prática educativa coletiva em seis dos treze serviços que referem a sua realização. Porém, independentemente do tipo de unidade, até no trabalho em grupos, a escuta e a conversa com as mulheres não fazem parte da atenção cotidiana.

Cerca de 48,9\% das gestantes - praticamente todas no PSF, 63,0\% nas Maternidades e 34,0\% nos Centros de Saúde - receberam algum tipo de orientação durante o pré-natal, particularmente sobre aleitamento materno, doenças sexualmente transmissíveis, câncer de colo uterino e cuidado com o bebê. Do total, 19,8\% foram instruídas sobre as doenças transmitidas pelo sexo, sendo maior no PSF $(45,4 \%)$, do que nas Maternidades $(28,3 \%)$ e Centros de Saúde (12,1\%) (Tabela 3). 
Orientações recebidas pelas gestantes durante o pré-natal, segundo o tipo de unidade. Recife, Pernambuco, Brasil, 1998.

\begin{tabular}{|c|c|c|c|c|c|c|c|c|}
\hline \multirow{3}{*}{ Orientação } & \multicolumn{8}{|c|}{ Tipo de unidade } \\
\hline & \multicolumn{2}{|c|}{$\begin{array}{c}C S \\
(n=232)\end{array}$} & \multicolumn{2}{|c|}{$\begin{array}{c}\text { MAT } \\
(n=46)\end{array}$} & \multicolumn{2}{|c|}{$\begin{array}{c}\text { PSF } \\
(n=55)\end{array}$} & \multicolumn{2}{|c|}{$\begin{array}{c}\text { Total } \\
\left(n=333^{*}\right)\end{array}$} \\
\hline & $\mathrm{n}$ & $\%$ & $n$ & $\%$ & $\mathrm{n}$ & $\%$ & $\mathrm{n}$ & $\%$ \\
\hline Recebeu orientação & 80 & 34,5 & 29 & 63,0 & 54 & 98,2 & 163 & 48,9 \\
\hline \multicolumn{9}{|l|}{ Orientações recebidas } \\
\hline Sintomas da gestação & 4 & 1,7 & 1 & 2,2 & 1 & 1,8 & 6 & 3,7 \\
\hline Cuidados com o próprio corpo & 9 & 3,9 & 1 & 2,2 & 14 & 25,4 & 24 & 14,7 \\
\hline Alimentação adequada & 27 & 11,6 & 10 & 21,7 & 13 & 23,6 & 50 & 30,7 \\
\hline Prevenção de câncer & 30 & 12,9 & 14 & 30,4 & 22 & 40,0 & 66 & 40,5 \\
\hline Doenças sexualmente transmissíveis & 28 & 12,1 & 13 & 28,3 & 25 & 45,4 & 66 & 40,5 \\
\hline Trabalho de parto & 16 & 6,9 & 4 & 8,7 & 17 & 30,9 & 37 & 22,7 \\
\hline Aleitamento materno & 35 & 15,1 & 16 & 34,8 & 38 & 69,1 & 89 & 54,6 \\
\hline Cuidados com o bebê & 21 & 9,0 & 13 & 28,3 & 31 & 56,4 & 65 & 39,9 \\
\hline Métodos para evitar filhos & 22 & 9,5 & 3 & 6,5 & 7 & 12,7 & 32 & 19,6 \\
\hline
\end{tabular}

CS = Centro de Saúde, MAT = Maternidade, PSF = Programa de Saúde da Família, *Sem informação: 22

À medida que aumenta o número de consultas realizadas no pré-natal, uma proporção significativamente maior de mulheres teve acesso a orientações ( $\mathrm{p}<0,001)$. Contudo, entre as grávidas com idade gestacional registrada, 38,0\% daquelas que estão no terceiro trimestre de gestação nunca foram orientadas.

Qualquer unidade de saúde pode solicitar o teste anti-HIV ao Laboratório Municipal de Saúde Pública, utilizando formulário específico. Este permite obter informações sobre o paciente (características sociodemográficas, condições clínicas e epidemiológicas), a amostra de sangue (data da coleta, local e condições) e o médico requisitante (nome e especialidade), a quem se enviará o resultado, em correspondência confidencial. Não há oferta universal deste teste nos serviços estudados e a equipe de saúde não foi capacitada para o aconselhamento. Além disso, excluídos os médicos da atenção tradi- cional, os seus componentes estão comprometidos com a execução de um amplo espectro de atividades.

Até mesmo as estratégias de testagem voltadas apenas para mulheres com risco identificado para infecção pelo HIV não têm sido devidamente executadas. Entre os profissionais que já reconheceram gestantes de risco para a AIDS $(64,3 \%)$, as principais condutas foram esclarecer sobre o diagnóstico $(37,0 \%)$, encaminhar para serviço de referência $(22,2 \%)$, para o COA $(18,5 \%)$ ou para consulta médica $(14,8 \%)$ e solicitar sorologia para HIV $(18,5 \%$ ) (Tabela 4). Nessas circunstâncias, só 26,7\% dos médicos requerem este teste, enquanto, 33,3\% encaminham as mulheres para que o exame seja requisitado no $\mathrm{COA}$ ou em outro serviço. Esta conduta cria dificuldades adicionais para as pacientes, constituindo-se em constante motivo de preocupação para a equipe do COA. 
Conduta dos profissionais frente à suspeita da gestante ser HIV positiva, segundo tipo de unidade. Recife, Pernambuco, Brasil, 1998.

\begin{tabular}{|c|c|c|c|c|c|c|c|c|}
\hline \multirow{3}{*}{ Condutas } & \multicolumn{8}{|c|}{ Tipo de unidade } \\
\hline & \multicolumn{2}{|c|}{$\begin{array}{c}\text { CS } \\
(n=28)\end{array}$} & \multicolumn{2}{|c|}{$\begin{array}{c}\text { MAT } \\
(n=4)\end{array}$} & \multicolumn{2}{|c|}{$\begin{array}{c}\text { PSF } \\
(n=10)\end{array}$} & \multicolumn{2}{|c|}{$\begin{array}{c}\text { Total } \\
(n=42)\end{array}$} \\
\hline & $\mathrm{n}$ & $\%$ & $\mathrm{n}$ & $\%$ & $\mathrm{n}$ & $\%$ & $\mathrm{n}$ & $\%$ \\
\hline Suspeito de soropositividade & 17 & 60,7 & 3 & 75,0 & 7 & 70,0 & 27 & 64,3 \\
\hline \multicolumn{9}{|l|}{ Condutas: } \\
\hline Orientar sobre o diagnóstico & 6 & 35,3 & - & - & 4 & 57,1 & 10 & 37,0 \\
\hline Encaminhar para serviço de referência & 3 & 17,6 & 1 & 33,3 & 2 & 28,6 & 6 & 22,2 \\
\hline Encaminhar para o COA & 3 & 17,6 & - & - & 2 & 28,6 & 5 & 18,5 \\
\hline Solicitar sorologia para HIV & 3 & 17,6 & 1 & 33,3 & 1 & 14,3 & 5 & 18,5 \\
\hline Encaminhar para consulta médica & 3 & 17,6 & - & - & 1 & 14,3 & 4 & 14,8 \\
\hline Orientar sobre a transmissão & 2 & 11,8 & 1 & 33,3 & - & - & 3 & 11,1 \\
\hline
\end{tabular}

CS = Centro de Saúde, MAT = Maternidade, PSF = Programa de Saúde da Família, COA = Centro de Orientação e Aconselhamento, HIV = Human Immunodeficiency Virus

Quando o médico solicita o exame, a amostra é coletada no próprio serviço, com a gestante concorrendo com a demanda geral de exames laboratoriais, sendo comum a ocorrência de rechaço. A produção do Laboratório Municipal de Saúde Pública que realizou, atendendo a rede básica de saúde, nos primeiros cinco meses de 1998, em média, 44 testes sorológicos da AIDS/mês, comprova a sua baixa solicitação. A equipe do laboratório tem constatado problemas no preenchimento da ficha de requisição da testagem - dados incompletos, ilegíveis — que impedem a avaliação dos casos e, muitas vezes, inviabiliza a própria devolução dos resultados.

Nos últimos dois anos, $57,1 \%$ dos 42 profissionais entrevistados receberam treinamento sobre doenças sexualmente transmissíveis/AIDS - 47,8\% dos médicos, 76,9\% das enfermeiras, uma das duas assistentes sociais e duas das quatro psicólogas. A maior proporção de profissionais treinados $(80 \%)$ foi encontrada no PSF - todas as enfermeiras e metade dos médicos - e nos Centros de Saúde $(57,1 \%)$. Nas Maternidades ninguém participou de treinamento. A maioria daqueles que não sentem necessidade de treinamento $(35,7 \%)$ atua nas Maternidades $(50 \%) \mathrm{e}$ nos Centros de Saúde (39,3\%).

Os médicos se mostram mais satisfeitos com o próprio conhecimento sobre a AIDS $(52,0 \%)$ e solicitam pouco uma revisão completa sobre o tema $(36,0 \%)$. Entretanto, são os únicos profissionais que se revelam interessados pela aquisição de conhecimentos sobre a doença em gestantes. As enfermeiras sentem uma maior carência de informações relativas ao tratamento da AIDS, enquanto as assistentes sociais e as psicólogas reivindicam uma revisão completa desses conteúdos.

\section{Discussão}

É marcante a vulnerabilidade institucional das ações de controle da transmissão vertical da AIDS, nas unidades avaliadas. Esta decorre de problemas organizacionais gerais, tais como os obstáculos para acesso ao atendimento, independentemente de ser primeira consulta ou seguimento, a desvalorização do trabalho educativo, a demanda reprimida para diagnóstico laboratorial e a falta de articulação entre as diversas atividades que compõem o conjunto das ações programáticas. Isso até mesmo quando se trata da prevenção de câncer cérvico-uterino — atividade fundamental para detectar os casos, sobretudo os assintomáticos, das doenças sexualmente transmissíveis e identificar a presença de infecções genitais que, aumentando a suscetibilidade ao HIV, podem interferir no parto e no puerpério. 14

Apesar das diferenças na organização do trabalho em saúde nos distintos tipos de atenção, o exame da operacionalidade do programa mostra que a execução do conjunto de atividades dirigidas à prevenção da transmissão materno-fetal do HIV dificilmente obedece à dinâmica processual estabelecida. Na efetivação da padronização normativa, é habitual 
constatar o não cumprimento da obrigatoriedade de informar todas as gestantes sobre o teste - respeitando o seu caráter voluntário e confidencial - o desconhecimento do fluxograma para diagnóstico sorológico da AIDS e a ausência de aconselhamento pré e pós-teste - falta capacitação às equipes para realizá-lo. Mesmo a estratégia de testagem voltada, apenas, para mulheres com risco identificado para infecção pelo vírus, encontra dificuldades operacionais na rede básica de saúde.

De modo genérico, embora no PSF uma maior proporção de mulheres tenha sido orientada, é pequena a valorização dos conteúdos relativos às doenças sexualmente transmissíveis/AIDS. A educação em saúde, utilizada como meio para a transmissão unilateral de informações, não tem se constituído em um instrumento capaz de promover o diálogo entre as usuárias e os profissionais de saúde. Além do mais, esta forma de atuar sobre o problema convive com a falta de percepção da vulnerabilidade pessoal frente à AIDS - dessas mulheres somente $11,1 \%$ fazem uso sistemático de preservativo ${ }^{15}-\mathrm{o}$ que torna ainda mais premente a necessidade da equipe de saúde assumir uma atitude iterativa, incorporando a participação das usuárias.

Entretanto, a insuficiência das respostas frente às necessidades de prevenção não está relacionada, apenas, ao gerenciamento das ações direcionadas à redução da transmissão do vírus da mãe infectada para seu filho. Não obstante os problemas na alocação, formação e supervisão da equipe de saúde, assim como, a falta de cumprimento dos papéis a serem exercidos por cada unidade, associada às deficiências na articulação formal entre os diferentes serviços e entre esses serviços e os agentes comunitários de saúde. Cabe examinar, também, algumas limitações da eleição da gravidez como o momento para detectar a infecção pelo HIV em mulheres.

No âmbito da prevenção, o cuidado com a gravidez pressupõe, também, o cuidado com a mu- lher e as suas práticas sexuais e de saúde, informando-a sobre as doenças sexualmente transmissíveis/ AIDS e fortalecendo-a para negociar com seu parceiro, antes que fique grávida e antes que seja infectada. Não é possível escamotear o fato de que a vulnerabilidade feminina à AIDS está intimamente relacionada à construção social do gênero, a qual, embora implique na opressão sobre homens e mulheres, ainda submete as mulheres a maiores restrições na expressão e satisfação das suas necessidades — ao menos quando se trata de estabelecer um acordo que viabilize a realização do sexo seguro por tempo prolongado. 16,17

Ao focalizar o pré-natal, esta formulação reforça uma organização do trabalho que dissocia as várias dimensões da saúde reprodutiva feminina - prevenção das doenças sexualmente transmissíveis, prevenção do câncer cérvico-uterino, assistência pré-natal e contracepção - acentua a verticalidade do modelo de atenção e mantém o caráter fragmentário e desarticulado das práticas de saúde. É fundamental que a racionalidade da ação pública reconheça que o amplo conjunto de necessidades de saúde das mulheres em idade fértil, rompendo com as fronteiras delimitadas pela gestação, reitera a importância da atenção integral.14,18,19

Neste sentido, a abordagem construída a partir da noção de vulnerabilidade reconhece a insuficiência das intervenções isoladas para produzir modificações importantes na saúde dos grupos populacionais e estimula o desenvolvimento de ações que contemplem um amplo conjunto de necessidades. Logo, fortalece um processo de trabalho fundado na complexa integração de ações individuais e coletivas, curativas e preventivas, articuladas em um sistema de cuidados em níveis progressivos de atuação.7,20 Desse modo, certamente, criam-se maiores condições para o atendimento das necessidades de prevenção da AIDS das mulheres e de seus filhos.

\section{Agradecimentos}

Às pesquisadoras de campo: Dionila Ferreira de Andrade, Maria das Dores Teixeira da Câmara e Suzette Maria Feitosa Brito. 


\section{Referências}

1. Vermelho LL, Silva LP, Costa ASL. Epidemiologia da transmissão vertical do HIV no Brasil. Bol Epidemiol 1999; 12: 5-15.

2. Recife. Secretaria de Saúde. Informe da Divisão de Epidemiologia e Vigilância Sanitária. Recife: Secretaria Municipal de Saúde; 2000.

3. Veloso VG, Vasconcelos AL, Grinsztein B. Prevenção da transmissão vertical no Brasil. Bol Epidemiol 1999; 12 16-25.

4. Ministério da Saúde. AIDS: recomendações para a prevenção da transmissão vertical do HIV. Brasília, DF: Ministério da Saúde; 1999.

5. Silva SLL. Cobertura e alguns aspectos qualitativos da assistência pré-natal de mães de crianças menores de 5 anos, no estado de Pernambuco [dissertação mestrado]. Recife: Instituto Materno Infantil de Pernambuco, IMIP; 2000.

6. Diniz S, Villela W. Interfaces entre os programas de DST/AIDS e Saúde da Mulher. In: Seminário AIDS e políticas de saúde da Associação Brasileira Interdisciplinar de AIDS; jan 1998; Rio de Janeiro, Rio de Janeiro.

7. Mann JM, Tarantola DJM, Netter TW. Assessing vulnerability to HIV infection and AIDS. In: Mann JM, Tarantola DJM, editors. AIDS in the world, the global AIDS policy coalition. Boston: Harvard University Press; 1992. p. 577-602.

8. Mann JM, Tarantola DJM. Vulnerability: personal and programmatic. In: Mann JM, Tarantola DJM, editors. AIDS in the world II - global dimensions, social roots, and responses. Boston: Harvard University Press; 1996. p. 4413.

9. Ayres JRCM, França-Júnior I, Calazans GJ, Saletti-Filho H. Vulnerabilidade e prevenção em tempos de AIDS. In: Barbosa RM, Parker R, organizadores. Sexualidades pelo avesso: direitos, identidades e poder. Rio de Janeiro: Instituto de Medicina Social (IMS) da Universidade Federal do Rio de Janeiro; 1999. p. 49-72.

10. Ayres JRCM. Vulnerabilidade e avaliação de ações preventivas. São Paulo: Casa de Edição; 1996.
11. Patton MQ. When to use qualitative methods. In: Patton MQ. How to use qualitative methods in evaluation. $3^{\text {th }}$ ed. California: Sage; 1989. p. 23-43.

12. Hartz ZMA. Pesquisa em avaliação da atenção básica: a necessária complementação do monitoramento. Divul Saúde Debate 2000; 26: 29-35.

13. Recife. Prefeitura. Guia de saúde. Recife: Secretaria Municipal de Saúde; 1996. p. 12-39.

14. D'Oliveira AFPL, Senna DM. Saúde da mulher. In: Schraiber LB, Mendes-Gonçalves RB, Nemes MIB, organizadores. Saúde do adulto, programas e ações na unidade básica. São Paulo: Hucitec; 1996. p. 86-108

15. Feliciano KVO, Kovacs, MH. Avaliação das ações de controle das DST/Aids desenvolvidas pela Secretaria $\mathrm{Mu}$ nicipal de Saúde da Cidade do Recife, com ênfase na atenção especializada e pré-natal: relatório final do projeto de avaliação. Recife: Secretaria Municipal de Saúde; 1998

16. Garcia SM. Conhecer os homens a partir do gênero e para além do gênero. In: Arilha M, Ridenti SGU, Medrado B, organizadores. Homens e masculinidades: outras palavras. São Paulo: Ecos; 1998. p. 31-50.

17. Paiva V. Fazendo arte com a camisinha. São Paulo: Summus; 2000. p.141-77.

18. Barbosa R, Lago TG. AIDS e direitos reprodutivos: para além da transmissão vertical. In: Parker R, organizador. Políticas, instituições e AIDS: enfrentando a epidemia no Brasil. Rio de Janeiro: Zahar; 1997. p. 163-75.

19. Villela W. A epidemia do HIV/AIDS entre as mulheres: um desafio para o Sistema Único de Saúde. In: Villela W, organizador. Mulher e AIDS: ambigüidades e contradições. São Paulo: Caderno NEPAIDS [Núcleo de Estudos de Prevenção da AIDS da Universidade de São Paulo] 1997; 41-7.

20. Schraiber LB, Mendes-Gonçalves RB. Necessidades de saúde e atenção primária. In: Schraiber LB, MendesGonçalves RB, Nemes MIB, organizadores. Saúde do adulto, programas e ações na unidade básica. São Paulo: Hucitec; 1996. p. 29-47.

Recebido em 10 de julho de 2002

Versão final reapresentada em 22 de julho de 2002

Aprovada em 31 de julho de 2002 